\title{
Structural and magnetic properties of inverse opal photonic crystals studied by x-ray diffraction, scanning electron microscopy, and small-angle neutron scattering
}

\author{
S. V. Grigoriev, ${ }^{1}$ K. S. Napolskii, ${ }^{2}$ N. A. Grigoryeva, ${ }^{3}$ A. V. Vasilieva, ${ }^{1}$ A. A. Mistonov, ${ }^{3}$ D. Yu. Chernyshov, ${ }^{4}$ \\ A. V. Petukhov, ${ }^{5}$ D. V. Belov, ${ }^{5}$ A. A. Eliseev, ${ }^{2}$ A. V. Lukashin, ${ }^{2}$ Yu. D. Tretyakov, ${ }^{2}$ A. S. Sinitskii, ${ }^{2}$ and H. Eckerlebe ${ }^{6}$ \\ ${ }^{1}$ Petersburg Nuclear Physics Institute, Gatchina, 188300 Saint-Petersburg, Russia \\ ${ }^{2}$ Department of Materials Science, M.V. Lomonosov Moscow State University, 119991 Moscow, Russia \\ ${ }^{3}$ Faculty of Physics, Saint-Petersburg State University, 198504 Saint-Petersburg, Russia \\ ${ }^{4}$ Swiss-Norwegian Beamlines, European Synchrotron Radiation Facility, 38000 Grenoble, France \\ ${ }^{5}$ van't Hoff laboratory, Debye Institute for Nanomaterials, Utrecht University, 3584 CH Utrecht, The Netherlands \\ ${ }^{6}$ GKSS Forschungszentrum, 21502 Geesthacht, Germany
}

(Received 12 October 2008; revised manuscript received 18 December 2008; published 27 January 2009)

\begin{abstract}
The structural and magnetic properties of nickel inverse opal photonic crystal have been studied by complementary experimental techniques, including scanning electron microscopy, wide-angle and small-angle diffraction of synchrotron radiation, and polarized neutrons. The sample was fabricated by electrochemical deposition of nickel in voids in a colloidal crystal film made of $450 \mathrm{~nm}$ polystyrene microspheres followed by their dissolving in toluene. The microradian small-angle diffraction of synchrotron radiation was used to reveal the opal-like large-scale ordering proving its tendency to the face-centered-cubic (fcc) structure with the lattice constant of $650 \pm 10 \mathrm{~nm}$. The wide-angle x-ray powder diffraction has shown that nanosize fcc nickel crystallites, which form an inverse opal framework, have some texture prescribed by principal directions in inverse opal on a macroscale, thus showing that the atomic and macroscopic structures are correlated. The polarized small-angle neutron scattering is used on the extreme limit of its ability to detect the transformation of the magnetic structure under applied field. Different contributions to the neutron scattering have been analyzed: the nonmagnetic (nuclear) one, the pure magnetic one, and the nuclear-magnetic interference. The latter in the diffraction pattern shows the degree of the spatial correlation between the magnetic and nuclear reflecting planes and gives the pattern behavior of the reversal magnetization process for these planes. The field dependence of pure magnetic contribution shows that the three-dimensional geometrical shape of the structure presumably leads to a complex distribution of the magnetization in the sample.
\end{abstract}

DOI: 10.1103/PhysRevB.79.045123

PACS number(s): 42.70.Qs, 61.05.fg, 61.05.cc, 81.07.-b

\section{INTRODUCTION}

Magnetic microstructured and nanostructured materials attract a growing attention, providing an immense field for fundamental physical experiments and a cornucopia of rapidly emerging applications, including magnetic tomography, magnetic hyperthermia, high-density magnetic data storage, magnetic logic elements, etc. ${ }^{1,2}$ This general statement is also applicable to a very specific class of such materialsmagnetic photonic crystals (PhCs). In general, $\mathrm{PhCs}$ are ordered structures with a periodic modulation of dielectric constant possessing photonic band gaps (PBGs). ${ }^{3,4}$ If made of materials with magnetic properties, $\mathrm{PhCs}$ could gain tunable magneto-optical properties and thus become promising for optical switching. ${ }^{5}$ A periodicity on a microscale can also make magnetic PhCs a unique test bench for the study of unusual magnetic phenomena, such as frustrated magnetism ${ }^{6}$ or giant magnetoresistance, ${ }^{7,8}$ discovered in ordered magnetic microstructures and nanostructures. However, routine fabrication of highly ordered three-dimensional (3D) magnetic $\mathrm{PhCs}$ with submicron periodicity remains a challenge.

A possible way toward magnetic PhCs employs selfassembly of monodisperse colloidal particles. Selfassembled colloidal crystals often referred to as artificial opals can act as magnetic PhCs with tunable optical properties if made of superparamagnetic particles ${ }^{9,10}$ or serve as templates for fabrication of magnetic inverse opals. In the latter case, the voids in colloidal crystals are filled with a targeted material and then colloidal particles are removed. ${ }^{11}$ Notorious examples of thus fabricated magnetic PhCs are inverse opals based on magnetic metals such as $\mathrm{Ni}, \mathrm{Co}, \mathrm{Fe}$, and their alloys. ${ }^{12-17}$ Besides, according to theoretical calculations, periodic metallic structures are of great interest as possible candidates for PhCs with complete PBGs in a visible range. ${ }^{18,19}$

Apparently, to observe microstructure-related magnetic and optical phenomena in metallic inverse opals, highquality samples are required. However, self-assembled materials typically possess numerous structural defects of different types, which is an important limiting factor to their use in optical devices. The local ordering in self-assembled PhCs is usually verified by scanning electron microscopy (SEM). Recently, laser diffraction was demonstrated to be effective for characterization of the structural quality of $\mathrm{PhCs}$ on a scale of up to several millimeters. ${ }^{20-22}$ However, the significant limitation of this method is that the diffraction can be observed only if the laser wavelength is less than $\mathrm{PhC}$ lattice period. Meanwhile, important PhCs for visible region possess a periodicity in a range of $400-500 \mathrm{~nm}$, making laser diffraction method not applicable for their study. A possible way to overcome this limitation relies on using different types of radiation with a wavelength short enough for recording diffraction patterns from $\mathrm{PhCs}$ of any periodicity. As we demonstrate in this work, $\mathrm{x}$-ray and neutron radiations can be successfully used for this purpose. 
We report on the synthesis and study of nickel inverse opal PhC. Despite the promising properties, which could be expected for metallic and magnetic $\mathrm{PhCs}$, we primarily considered nickel inverse opal as a complex structure exhibiting a unique interplay of structural and magnetic properties. The sample has been studied on the macroscopic and microscopic levels by complementary experimental techniques, including SEM, small- and wide-angle diffractions of synchrotron radiation, and small-angle neutron scattering (SANS). Interestingly, although SANS is extensively used for the study of spatially ordered materials, ${ }^{23}$ to the best of our knowledge, it was never applied to the characterization of inverse opal PhCs. Furthermore, we used polarized SANS to study microstructure-dependent magnetic properties of $\mathrm{Ni}$ inverse opal, since this method is sensitive both to the nuclear and magnetic structures.

The paper is organized in the following way. Section II gives the essence of the sample preparation procedure and description of the experimental techniques. Section III describes the microradian and wide-angle synchrotron diffraction giving the structural features of $\mathrm{PhC}$ on different levels. The results of the polarized SANS experiments are presented in Sec. IV. Section V gives short concluding remarks.

\section{SAMPLE AND EXPERIMENTAL}

The Ni PhC films with an inverse opal structure were prepared using a templating technique. Colloidal crystal films made of $450 \mathrm{~nm}$ polystyrene microspheres were grown onto polished $\mathrm{Cu}$ substrates by the vertical deposition method. Electrochemical crystallization of nickel in the voids between the spheres was carried out in three-electrode cell at room temperature. The counter electrode was a $\mathrm{Pt}$ wire and the reference electrode-saturated $\mathrm{Ag} / \mathrm{AgCl}$ electrode connected to the cell via a Luggin capillary; $0.1 \mathrm{M} \mathrm{NiCl}_{2}, 0.6$ $\mathrm{M} \mathrm{NiSO}_{4}, 0.3 \mathrm{M} \mathrm{H}_{3} \mathrm{BO}_{3}$, and $3.5 \mathrm{M} \mathrm{C}_{2} \mathrm{H}_{5} \mathrm{OH}$ solution was used for potentiostatic Ni deposition at $E_{d}=-0.85 \mathrm{~V}$ versus reference electrode. In order to obtain free-standing Ni structure onto $\mathrm{Cu}$ substrate, the polystyrene microspheres were dissolved in toluene. Finally the sample was a film with an area of $5 \times 10 \mathrm{~mm}^{2}$ and a thickness of $12 \mu \mathrm{m}$.

Examples of the SEM images of the sample (recorded by LEO Supra 50 VP instrument) are shown in Figs. 1(a) and 1(b). The image shown in Fig. 1(a) can be attributed to either the (111) plane of the face-centered-cubic (fcc) structure with hexagonal layers interchanging as $(A B C A B C)$ or to the base plane of the random hexagonal-close-packing (RHCP) structure, which is often observed in colloid self-assembly. ${ }^{24-28,31}$ The cross-section image in Fig. 1(b) made after rotation of the sample by $55^{\circ}$ shows that at this particular place in the sample, the structure is cubically ordered and the image can be attributed to the (200) plane. However, the SEM method is suffering from its locality and the surface image does not allow one to make a conclusion on the internal structure of the sample.

To address different aspects of the sample structure and its magnetic ordering, we used the different radiation sources (neutron or synchrotron) and changed the distance between the sample and the detector to get the appropriate values of

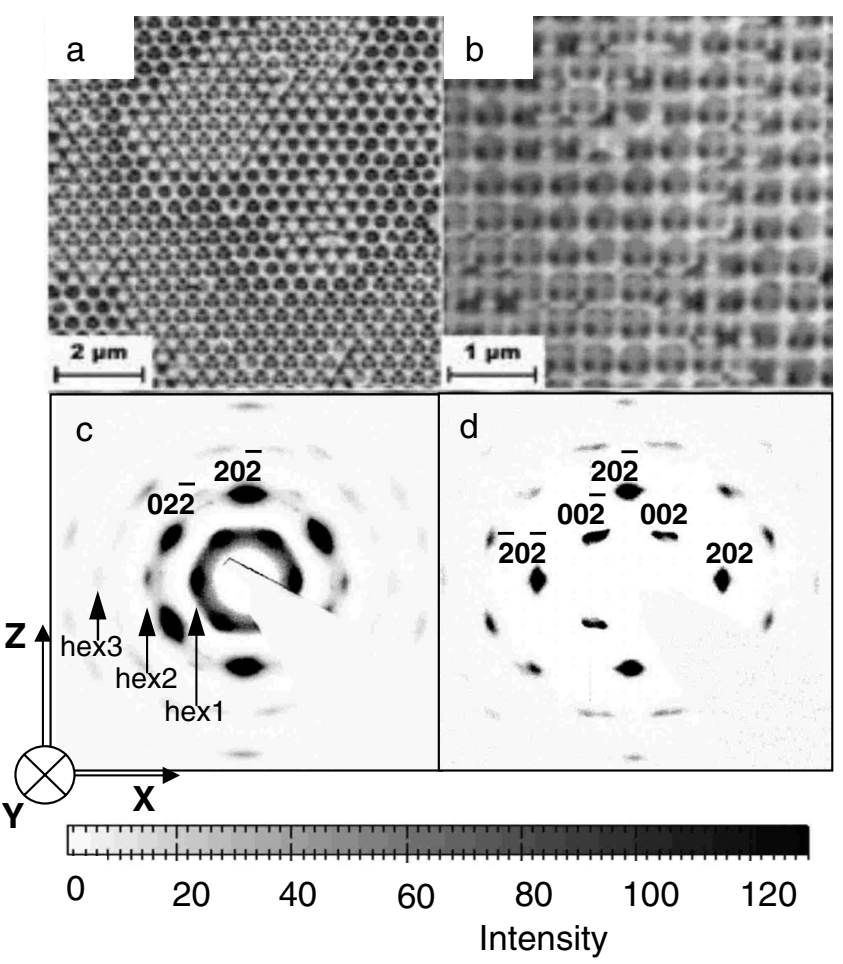

FIG. 1. SEM images of (a) the top and (b) side views of $\mathrm{Ni}$ inverse opal. Small-angle synchrotron diffraction patterns measured at (c) the normal incidence and after (d) the sample rotation by $55^{\circ}$.

the scattering angles: small angles for the large scale structure and wide angles for the atomic structure.

The mesoscopic structure of the sample is studied with synchrotron radiation using the microradian setup [Fig. 2(a)], similar to the one described in Refs. 29 and 30, at the DutchBelgian beam line ("DUBBLE") BM26 (ESRF, Grenoble, France). The sample has been mounted on a goniometer, which allows for sample translation and for careful sample orientation around two axes orthogonal to the beam. Particularly, the rotation around the vertical axis can be done for the wide range of angles $\omega$ (Fig. 2). Diffraction of a $13 \mathrm{keV}$ x-ray beam (wavelength $\lambda=0.95 \AA$, bandpass $\Delta \lambda / \lambda=2$ $\times 10^{-4}$, and size at the sample about $0.5 \times 0.5 \mathrm{~mm}^{2}$ ) is registered at $8 \mathrm{~m}$ distance by a two-dimensional charge-coupled

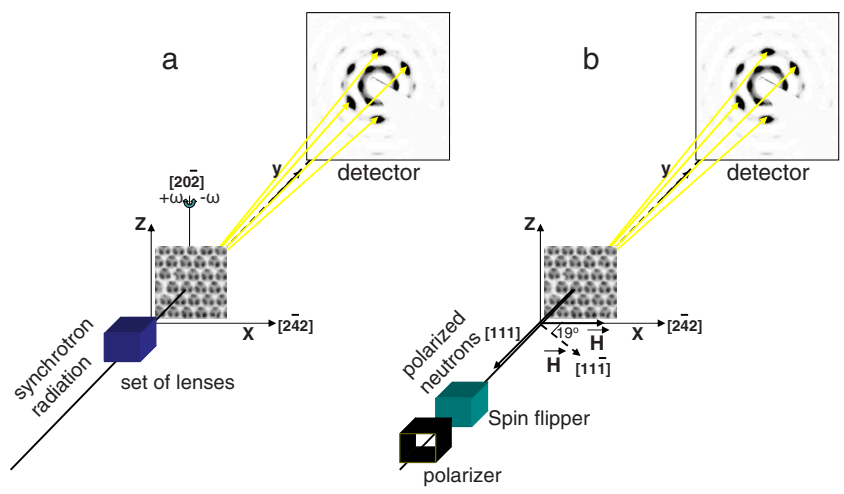

FIG. 2. (Color online) Schematic drawing of the diffraction experiments with (a) the use of synchrotron radiation and (b) the use of polarized neutrons. 
device detector (Photonic Science, $4008 \times 2671$ pixels of 22 micronsquare). To improve the resolution, the beam was focused by a set of compound refractive lenses installed in front of the sample. This setup allows achieving angular resolution of the order of a few microradians, which is sufficient for collecting detailed information on the structure of largeperiod photonic crystals. ${ }^{29,30}$

The polarized SANS experiments were carried out at the SANS-2 scattering facility of the FRG-1 research reactor in Geesthacht (Germany). Figure 2(b) presents the schematic outline of SAS experiment. A polarized beam of neutrons with an initial polarization $P_{0}=0.95$, a neutron wavelength $\lambda=1.2 \mathrm{~nm}$, a bandwidth $\Delta \lambda / \lambda=0.1$, and a divergence $\eta$ $=1.5 \mathrm{mrad}$ was used. The scattered neutrons were detected by a position sensitive detector with a resolution of 256 $\times 256$ pixels; each of $2.2 \times 2.2 \mathrm{~mm}^{2}$ size. The detectorsample distance was set at its maximum of $21.5 \mathrm{~m}$ such that the $q$ range was covered from 0.005 to $0.07 \mathrm{~nm}^{-1}$ with a step of $0.0005 \mathrm{~nm}^{-1}$. The external magnetic field $\mathbf{H}$ from 1 to $150 \mathrm{mT}$ was applied perpendicular to the incident beam and along the crystallographic [2 $\overline{4} 2]$ axis of the $\mathrm{PhC}$ or by angle

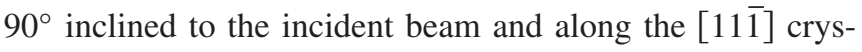
tallographic axis of the $\mathrm{PhC}$. The neutron polarization always followed the direction of the external magnetic field. The scattered intensity can be measured in the experiments with the incident beam whose polarization is parallel $\left(+P_{0}\right.$ $\left.=+\mathbf{P}_{0} \mathbf{h}\right)$ or antiparallel $\left(-P_{0}=-\mathbf{P}_{0} \mathbf{h}\right)$ to the external magnetic field $\mathbf{h}$. The orientation of the polarization with respect to the magnetic field can be automatically changed by the spin flipper [Fig. 2(b)].

\section{STRUCTURE OF THE INVERSE OPAL PHOTONIC CRYSTAL}

\section{A. Mesoscopic periodic structure}

The microradian x-ray diffraction (XRD) with synchrotron radiation is known to be powerful in determining the structure and long-range ordering of photonic crystals. $^{27,28,31-33}$ Figure 1(c) displays the X-ray small-angle diffraction pattern measured with the beam orthogonal to sample surface. The hexagonal arrangement of the Bragg reflections reveals the hexagonal ordering within the planes parallel to the surface. The strongest reflections can be understood assuming a fcc structure with the lattice constant of $650 \pm 10 \mathrm{~nm}$. In Fig. 1(c) we give the $h k l$ assignment to some of them within the fcc basis frame. However, one can also see a few additional weaker reflections, which are not allowed for an fcc structure. Their origin can be related rather to the finite thickness than to an RHCP structure of the crystal.

To prove that fcc is the dominating crystal structure on the macroscopic scale, diffraction patterns were recorded at various spots on the sample surface and at various sample orientation. We varied the angle $\omega$ around the [20̄] axis from $\omega=-60^{\circ}$ to $\omega=+60^{\circ}$ [Fig. 2(a)]. Figure $1(\mathrm{~d})$ presents a pattern measured at $\omega=54.7^{\circ}$. In this case the x-ray beam propagates along the [020] axis of the fcc structure. The pattern, indeed, reveals the fourfold symmetry of the (020) crystallo-

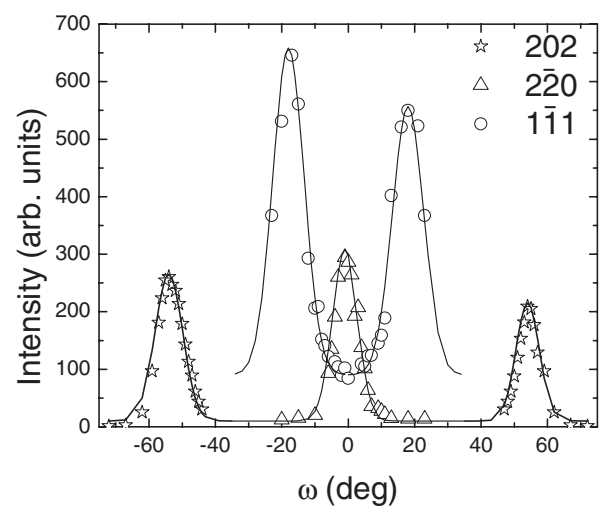

FIG. 3. The $\omega$ dependence of the intensity of the [202], [220], and $[1 \overline{1} 1]$ reflections.

graphic planes. The $\omega$ scans for the various reflections (202), $(2 \overline{2} 0)$, and $(1 \overline{1} 1)$ during sample rotation are summarized in Fig. 3. The intensity of the $(2 \overline{2} 0)$ reflection is maximal at $\omega=0$. The maxima of the $(1 \overline{1} 1)$ reflection and (202) reflection arise at $\omega= \pm 19.5^{\circ}$ and at $\omega= \pm 54.7^{\circ}$, respectively. The mosaic of the structure taken over these reflections is of order $5^{\circ}-7^{\circ}$. Interestingly that the maxima of almost equal intensities are recorded at both positive and negative sides of the $\omega$ scan. This observation signifies the disorder of the fcc structure attributed to the twinning of the domains with the $\ldots A B C A B C \ldots$ and $\ldots A C B A C B \ldots$ stacking type. The ratio between their volumes is estimated as 4:5.

\section{B. Atomic periodic structure}

The wide-angle powder x-ray diffraction experiment was performed on the same $\mathrm{PhC}$ using the MAR diffractometer at the BM01A beam line (SNBL) at ESRF with wavelength $\lambda$ $=0.71668 \AA$. At the incident beam normal to the surface of the $\mathrm{PhC}$, the scattering pattern gives an example of typical powder diffraction, i.e., a system of concentrated rings of an equal intensity. It, therefore, shows no presence of any texture in the plane of the PhC film. The positions of the reflexes can be prescribed to the fcc atomic structure of $\mathrm{Ni}$

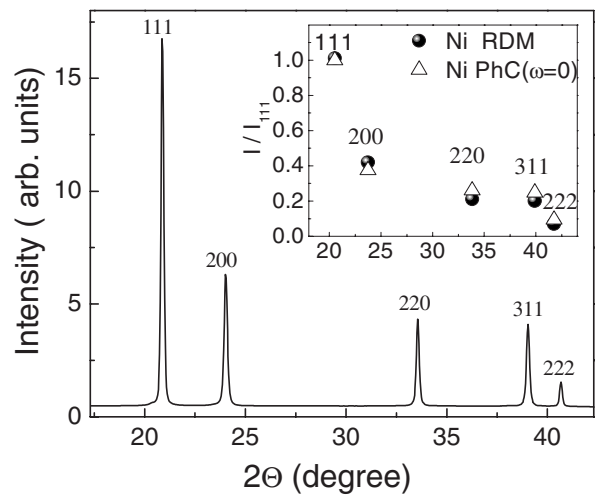

FIG. 4. X-ray powder-diffraction data for the Ni inverse opal photonic crystal. The inset shows the comparison of the intensities of the reflections normalized to that of the (111) peak for the randomly oriented $\mathrm{Ni}$ powder and for the sample under study at $\omega=0$. 


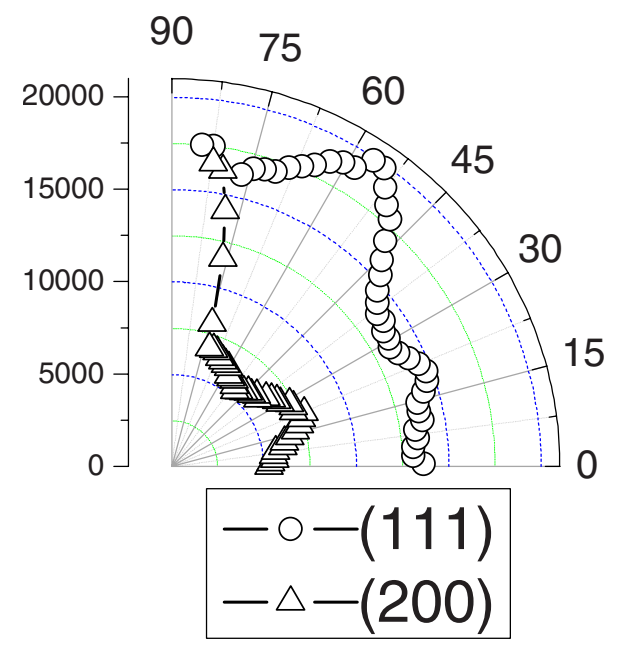

FIG. 5. (Color online) $\omega$ scan of the [111] and [200] reflections of fcc Ni structure, revealing the out-plane texture of the $\mathrm{PhC}$. The atomic planes (111) and (200) of Ni crystallites are inhomogeneously distributed over the $\mathrm{PhC}$.

with lattice constant $a_{0}=3.52 \AA$ (Fig. 4). The intensities of diffraction peaks normalized to that of the (111) peak are presented in the inset in Fig. 4. The relative intensities of the randomly oriented crystallites of Ni powder are shown in the same figure for comparison. As can be seen from the figure for a given orientation of the $\mathrm{PhC}$, the scattering intensities from the planes (200), (220), (311), and (222) are close to those of the corresponding peaks for the randomly oriented powder sample. The preliminary conclusion is that the $\mathrm{Ni}$ crystallites of the fcc structure are randomly oriented in the plane of the PhC but they may have some out-plane texture.

The intensities of the [111] and [200] reflections taken in the $\omega$ scan are shown in Fig. 5. They give the distribution of the (111) and (200) atomic Ni planes. Here the axis with $\omega$ $=0^{\circ}$ corresponds to the direction normal to the $\mathrm{PhC}$ film (the [111] axis of the $\mathrm{PhC}$ ) and the axis with $\omega=90^{\circ}$ lies in the plane of the $\mathrm{PhC}$ film. The experimental points at $\omega$ close to $90^{\circ}$ are excluded from the data set because of the large absorption of the beam transmitting through the film in this geometry. As is well seen, the curve for the (111) planes has two maxima at $\omega \approx 55^{\circ}$ and $\omega \approx 20^{\circ}$. The curve for the (200) planes has its maxima at $\omega \approx 20^{\circ}$ and, presumably, at $\omega$ $\approx 90^{\circ}$. The more pronounced maxima at $90^{\circ}$ for the (200) planes and at $55^{\circ}$ for the (111) planes are obviously interconnected. They stem from the same atomic Ni crystallites, which are disposed by their faces on the $\mathrm{PhC}$ plane. The less pronounced maxima at $20^{\circ}$ both for (111) and (200) planes show that they prefer equally the orientation parallel to the system of $\langle 111\rangle$ axes of the $\mathrm{PhC}$, which are $20^{\circ}$ inclined to the sample plane. The considerable amount of the crystallites is randomly oriented over the inverse PhC. From the ratio between two integral intensities for the randomly oriented crystallites and the crystallites with the preferable orientation, one can estimate that most of the Ni crystallites are randomly oriented but from 10 to $15 \%$ of its amount prefer (200) planes of Ni crystallites parallel to the PhC surface.

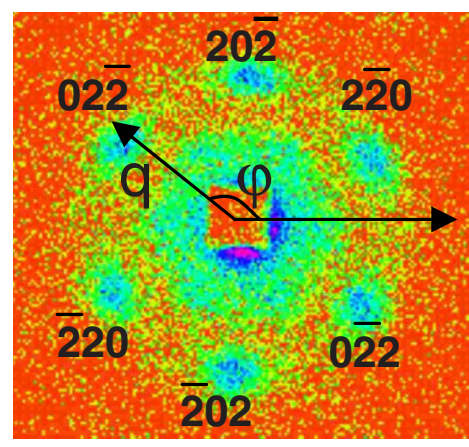

FIG. 6. (Color online) The neutron-scattering map for Ni inverse opal photonic crystal.

\section{MESOSCOPIC MAGNETIC STRUCTURE}

In SANS measurements, we recorded the neutrondiffraction patterns consisted of several clearly resolved sets of hexagonally arranged reflexes as shown in Fig. 6. The neutron-scattering intensities $I\left(\mathbf{q},+P_{0}\right)$ and $I\left(\mathbf{q},-P_{0}\right)$ were measured in the experiments with the neutron beam whose polarization was parallel and antiparallel to the external magnetic field, respectively. Further on, it is supposed that the nuclear and magnetic structures with a very large period are characterized by the same structure factor $\mathcal{S}(q)$ and the same form factor $F(\mathbf{q})$ as a result of scattering from the same base element of this structure. Polarization-independent part of the scattering $\left[I(q)=I\left(\mathbf{q},+P_{0}\right)+I\left(\mathbf{q},-P_{0}\right)\right]$ represents the sum of the nuclear and magnetic scattering,

$$
I(\mathbf{q})=I_{N}+I_{M} \sim\left|A_{n} \mathcal{S}(q) F(\mathbf{q})\right|^{2}+\left|A_{m} \mathbf{m}_{\perp \mathbf{q}} \mathcal{S}(q) F(\mathbf{q})\right|^{2} .
$$

The nuclear contribution $I_{N}$ to the peak intensity is obtained for nonmagnetized sample $(\langle m\rangle=0)$; in that case the magnetic coherent scattering vanishes and the diffuse small-angle scattering arises. For the pure magnetic coherent part one gets

$$
I_{M}(\mathbf{q})=I[\mathbf{q}, m(H)]-I(\mathbf{q}, 0) \sim\left|A_{m} \mathbf{m}_{\perp \mathbf{q}} \mathcal{S}(q) F(\mathbf{q})\right|^{2} .
$$

The polarization-dependent part of the scattering $[\Delta I(\mathbf{q})$ $\left.=I\left(\mathbf{q},+P_{0}\right)-I\left(\mathbf{q},-P_{0}\right)\right]$ is attributed to the nuclear-magnetic interference indicating the correlation between the magnetic and nuclear structures,

$$
\Delta I(\mathbf{q}) \sim 2\left(\mathbf{P}_{0}\langle\mathbf{m}\rangle_{\perp \mathbf{q}}\right) A_{n} A_{m}|\mathcal{S}(q) F(\mathbf{q})|^{2},
$$

where $\mathbf{m}$ and $\hat{\mathbf{q}}$ are the unit vectors of magnetization $\mathbf{M}$ and the momentum transfer $\mathbf{q}$, respectively, and $\mathbf{m}_{\perp \mathbf{q}}=\mathbf{m}$ $-(\hat{\mathbf{q} m}) \hat{\mathbf{q}}$. To analyze the interference scattering, it is convenient to define "polarization" in the form

$$
P_{s}=\frac{\Delta I(q)}{I(q)}=2\left(\left\langle\mathbf{P}_{0}\right\rangle\langle\mathbf{m}\rangle_{\perp \mathbf{q}}\right) \frac{A_{m}}{A_{n}} \approx 2 P_{0} \frac{p_{\mathrm{Ni}}}{b_{\mathrm{Ni}}} \sin ^{2} \varphi .
$$

Two important features should be noted. First, the intensity of different contributions is determined by the amplitudes of the nuclear $A_{n}=b_{\mathrm{Ni}} N_{0}$ and magnetic $A_{m}=p_{\mathrm{Ni}} N_{0}$ scatterings. Here $b_{\mathrm{Ni}}=1.03 \times 10^{-12} \mathrm{~cm}$ is the coherent scattering length of $\mathrm{Ni}$ nuclei, $p_{\mathrm{Ni}}=0.16 \times 10^{-12} \mathrm{~cm}$ is the coherent magnetic scattering length of $\mathrm{Ni}$ atom in the small-angle 

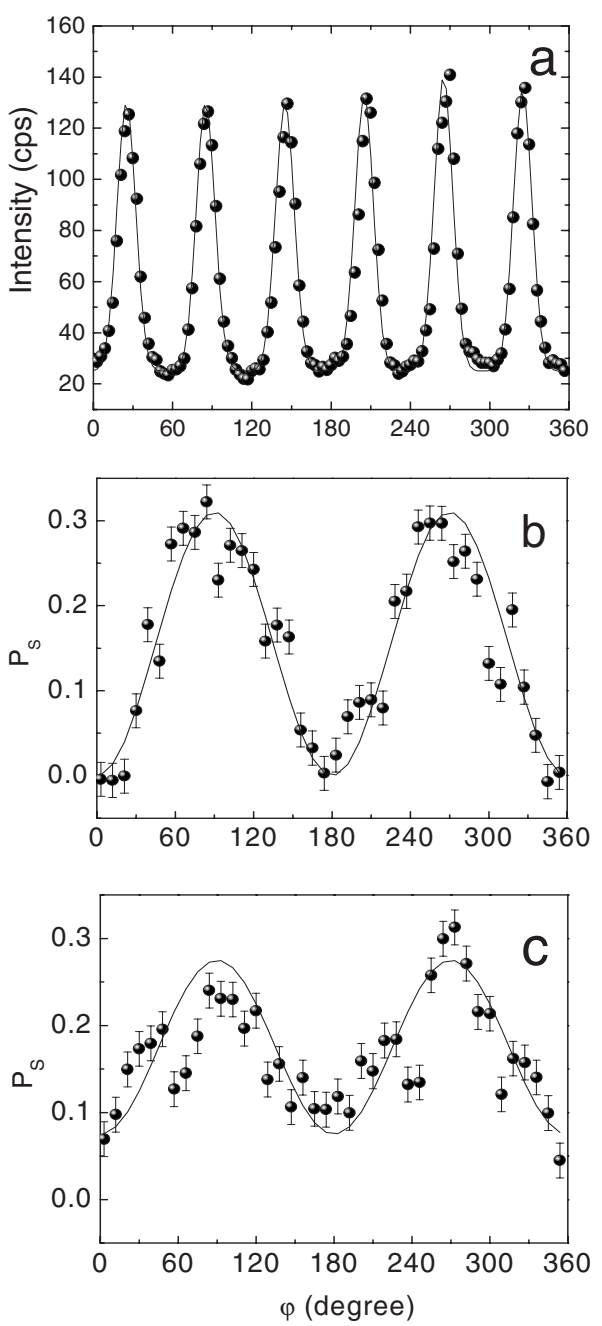

FIG. 7. (a) The $\varphi$ dependence of the intensity and polarization for the family of $\langle 202\rangle$ reflections for the external magnetic field

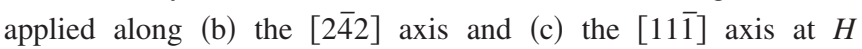
$=150 \mathrm{mT}$.

range, and $N_{0}$ is the atom density. One estimates from these numbers $\left[I_{M} / I_{N}=\left(p_{\mathrm{Ni}} / b_{\mathrm{Ni}}\right)^{2}=0.024\right]$ that the intensity of nuclear scattering is 2 orders of magnitude bigger than that of the magnetic scattering for the sample under study. The estimated polarization can be much larger: $P_{s} \approx 0.3$. Taking into account the scattering volume of the sample and the high requirements to the resolution leading to low intensity of the incident beam, one can predict that the interference contribution is well recordable while the pure magnetic contribution is hard to distinguish on the background of the nuclear one. Second, the small-angle diffuse scattering should be added to the diffraction reflections due to structure imperfection and particle size dispersion. It appears also when a magnetic structure is broken up into domains at $H$ $\sim H_{c}$.

To adequately take into consideration the magnetic and interference scattering, we perform the analysis of intensities as a function of the azimuth angle $\varphi$, where $\varphi$ is the angle between the direction of the scattering vector $\mathbf{q}$ and the horizon in the detector plane (Fig. 6). Figure 7(a) shows the dependence of the intensity $I(q, \varphi)$ at $q=q_{202}$ on the angle $\varphi$
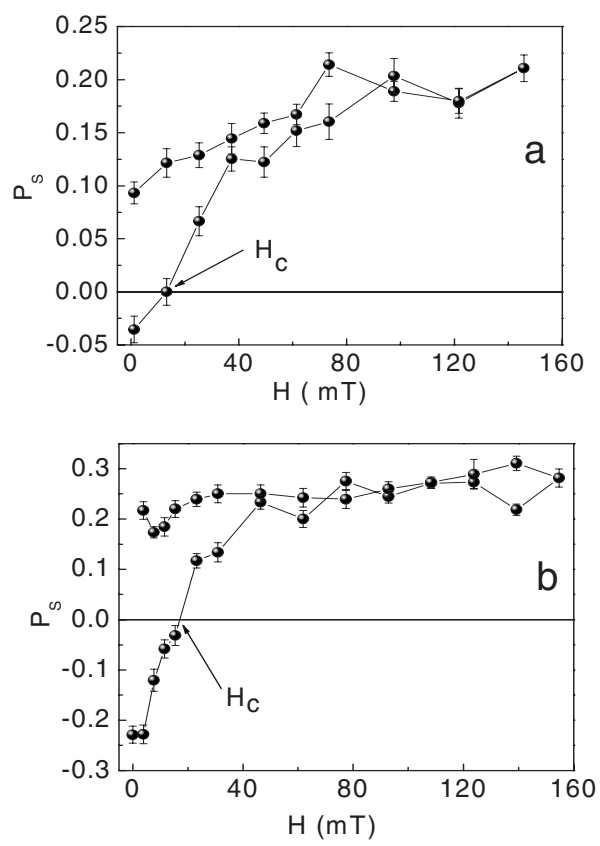

FIG. 8. The field dependence of the polarization $P_{s}$ at the scattering vector $\mathbf{q} \|[20 \overline{2}]$ for (a) the field $\mathbf{H} \|[2 \overline{4} 2]$ and (b) the field $\mathbf{H} \|[1 \overline{1} \overline{1}]$.

for a magnetized sample $(H=150 \mathrm{mT})$. Six clearly pronounced maxima at $60^{\circ}$ intervals can be seen; they correspond to six $(20 \overline{2})$ reflections. Figures $7(\mathrm{~b})$ and 7 (c) show the $\varphi$ dependence of the polarization $P_{s}$ at $q=q_{20 \overline{2}}$, i.e., for the family of the $\langle 20 \overline{2}\rangle$ reflections and for two orientations of the external magnetic field.

As can be seen $P_{S}$ is adequately described by a function of the form $\sin ^{2} \varphi$ with an estimated value of $p_{\mathrm{Ni}} / b_{\mathrm{Ni}}=0.14$, which is consistent with a value of 0.155 predicted from the theory of scattering. The function of this form suggests that the family of the planes (202) was equally magnetized along the external field direction at this value of the field $H$ $=150 \mathrm{mT}$. In case the field is directed in the plane of the sample $(\mathbf{H} \|\langle 2 \overline{4} 2\rangle)$, the polarization is zero at $\mathbf{q} \|\langle 2 \overline{4} 2\rangle$. If the field is directed along the $\langle 11 \overline{1}\rangle$ axis then the polarization is positive at $\mathbf{q} \|\langle 2 \overline{4} 2\rangle$. The variations in the polarization with the azimuth angle $\varphi$ for the two different orientations of the magnetic field can be well interpreted taking into account the geometry of the experiment, i.e., mutual orientation of three vectors: the field and polarization axes $(\mathbf{H} \| \mathbf{P})$, the average magnetization vector $\langle\mathbf{m}\rangle$, and scattering vector $\mathbf{q}$. The occurrence of the polarization (interference scattering) at the same values of $q$, as for nuclear scattering, suggests that the magnetic and nuclear structures coincide.

The magnetic field dependence of the polarization $P_{S}$ for $q \|[20 \overline{2}]$ is shown in Figs. 8(a) and 8(b) for the field directed

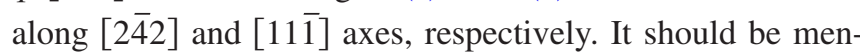
tioned that the observed polarization $P_{S}$ is proportional to the average magnetization $\left(P_{s} \sim\langle m\rangle\right)$ projected onto the magnetic field direction [Eq. (4)]. As can be seen from Fig. 8 the saturation of the magnetization $\langle m\rangle$ takes place at $60 \mathrm{mT}$ for $\mathbf{H} \|[2 \overline{4} 2]$ and at $40 \mathrm{mT}$ for $\mathbf{H} \|[11 \overline{1}]$. The magnetization 

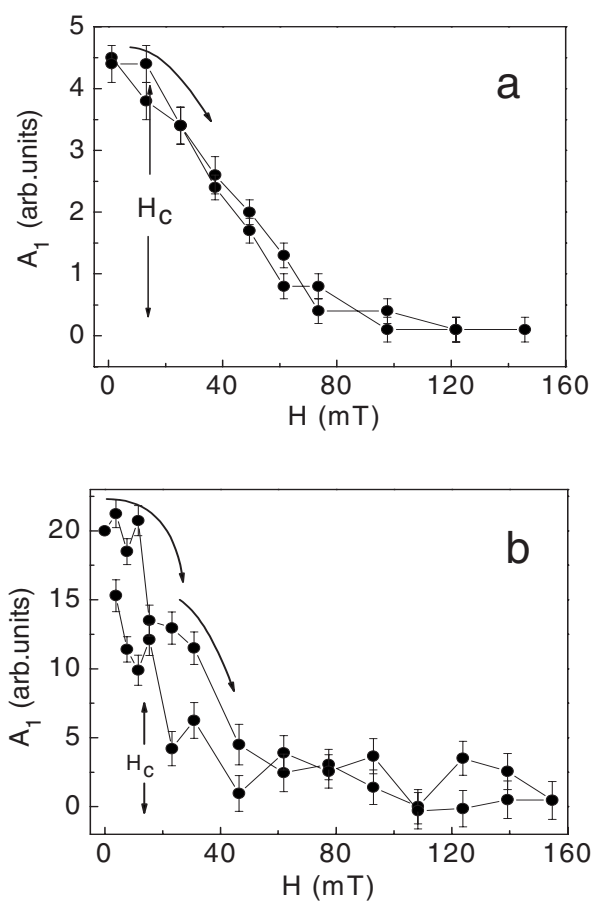

FIG. 9. The field dependence of the amplitude $A_{1}$ of the smallangle diffuse scattering intensity at (a) $\mathbf{H} \|[2 \overline{4} 2]$ and (b) at $\mathbf{H} \|[11 \overline{1}]$.

curve shows also the hysteretic behavior with the coercive force $H_{c}=15 \pm 2 \mathrm{mT}$ for $\mathbf{H} \|[2 \overline{4} 2]$ and $H_{c}=20 \pm 2 \mathrm{mT}$ for $\mathbf{H} \|[11 \overline{1}]$. The remnant magnetization $\langle m\rangle$ is twice higher for $\mathbf{H} \|[11 \overline{1}]$ than for $\mathbf{H} \|[2 \overline{4} 2]$. Thus, for $\mathbf{H} \|[11 \overline{1}]$ the hysteresis loop is much more rectangular than for $\mathbf{H} \|[2 \overline{4} 2]$. This observation implies that the [11]] direction is the axis easier for magnetization as compared with the [2 $\overline{4} 2]$ axis that is related to the geometrical shape of the inverse opal photonic crystal. The field dependence of the polarization $P_{s}$ for other reflections $\langle 202\rangle$ shows the similar behavior as described above.

The pure magnetic scattering $I_{H}(q)$ has been analyzed at different scattering vectors $\mathbf{q} \|[20 \overline{2}]$ and $\mathbf{q} \|[2 \overline{2} 0]$. We suggested in Eq. (2) that the magnetic scattering $I_{H}(q)$ is a difference between the magnetic cross sections of the sample in two principally different states: partially magnetized in the finite field $H$ and fully demagnetized at $H=H_{c}$. The main component of the magnetic cross section at large fields is a system of the magnetic reflections described as the sum of Gaussians with positions corresponding to the nuclear maxima. On contrary, the major component of the cross section at $H=H_{c}$ is the diffuse scattering from the domains, which can be described by the squared Lorenzian in the absence of any contribution of the reflections. The experimental data were treated in accord to this model. The amplitude of the diffuse scattering $A_{1}$, describing the scattering from magnetic domains, is shown in Fig. 9. It is maximal at $H$ $<H_{c}$ upon descending field and decreases with the field, while it does not vanish at $H=80 \mathrm{mT}$ for $\mathbf{H} \|[2 \overline{4} 2]$ and at $H=40 \mathrm{mT}$ for $\mathbf{H} \|[11 \overline{1}]$. Thus, domains disappear at smaller values of $H$ for $\mathbf{H} \|[11 \overline{1}]$ than for $\mathbf{H} \|[2 \overline{4} 2]$, again demon-
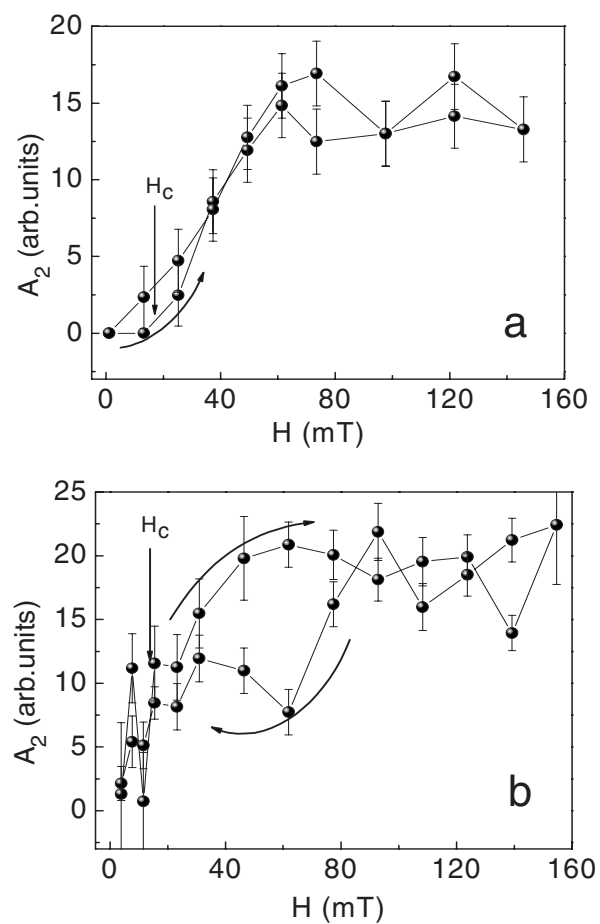

FIG. 10. The field dependence of the Bragg intensity at $\mathbf{q} \|[20 \overline{2}]$ for (a) $\mathbf{H} \|[2 \overline{4} 2]$ and (b) $\mathbf{H} \|[11 \overline{1}]$.

strating that the sample is easier to magnetize in the [11 $\overline{1}]$ direction than in the [2 $\overline{4} 2]$ direction.

All reflections of $\langle 202\rangle$ type visible in Fig. 6 can be divided into two subgroups: reflections $(20 \overline{2})$ and $(\overline{2} 02)$ with the angle of $90^{\circ}$ between $\mathbf{q}$ and $\mathbf{H}$ and reflections $(02 \overline{2})$, $(0 \overline{2} 2),(2 \overline{2} 0)$, and $(\overline{2} 20)$ with the angle of about $30^{\circ}$ between $\mathbf{q}$ and $\mathbf{H}$. To improve the statistics, the intensities of these magnetic reflections were averaged over the subgroup. Figure 10 shows the intensity of the $(20 \overline{2})$ reflection as a function of the applied magnetic field for $H \|[2 \overline{4} 2]$ (a) and for $H \|[11 \overline{1}](\mathrm{b})$, respectively. The intensity of the reflection is zero at $H \sim H_{c}$ then it increases and reaches the saturation values at $H=60 \mathrm{mT}$ for $\mathbf{H} \|[2 \overline{4} 2]$ and at $H=40 \mathrm{mT}$ for $\mathbf{H} \|[11 \overline{1}]$. The dependence for $\mathbf{H} \|[2 \overline{4} 2]$ shows no hysteretic behavior within error bars, but for $\mathbf{H} \|[11 \overline{1}]$ the hysteresis is observed in the field range from 35 to $80 \mathrm{mT}$; although it can be also related to the poor statistics of the pure magnetic scattering.

An interesting behavior of the scattering intensities is observed at the reflection $(02 \overline{2})$. They are shown in Fig. 11 as a function of the field for $\mathbf{H} \|[2 \overline{4} 2]$ (a) and for $\mathbf{H} \|[11 \overline{1}]$ (b), respectively. As for the previous case, the scattering intensity is zero at $H_{c}$ and saturates at large fields $H>100 \mathrm{mT}$. On the other hand, the curves show several features. For $\mathbf{H} \|[2 \overline{4} 2]$ (i) there are two critical fields. The first one at $H_{C 1}=20 \mathrm{mT}$, below this value the intensity is zero. It means that the corresponding planes $\{02 \overline{2}\}$ are not magnetized. Above $H_{C 2}=60 \mathrm{mT}$ the intensity is saturated showing full magnetization alignment along the field inside these planes. 

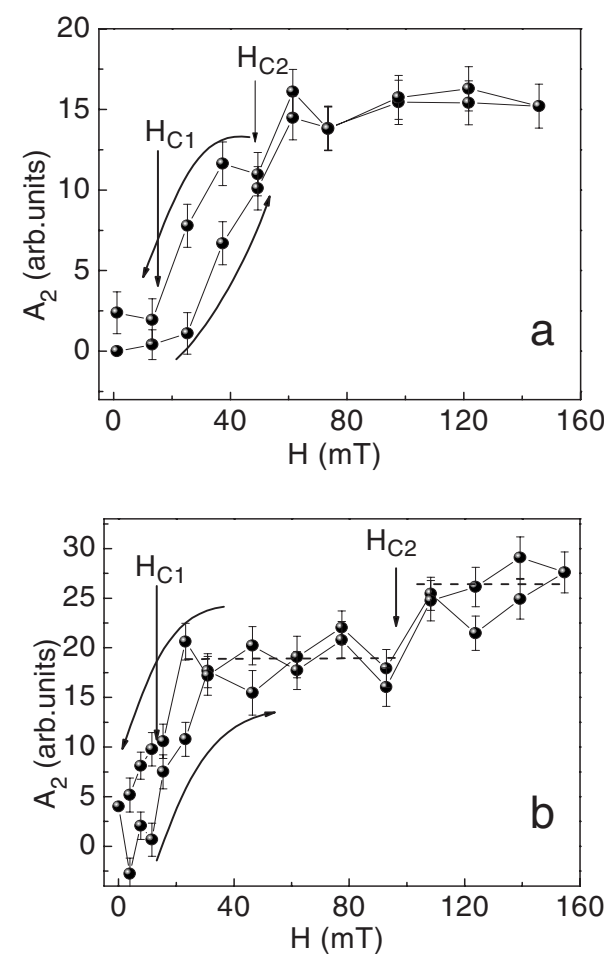

FIG. 11. The field dependence of the Bragg intensity at $\mathbf{q} \|[2 \overline{2} 0]$ for (a) $\mathbf{H} \|[2 \overline{4} 2]$ and (b) $\mathbf{H} \|[11 \overline{1}]$.

(ii) There is a small hysteresis loop inside the field range between two critical fields $H_{C 1}<H<H_{C 2}$. For $\mathbf{H} \|[11 \overline{1}]$ there are two critical fields as well. The $H$ dependence looks a steplike function with $H_{C 1}=20 \mathrm{mT}$ and $H_{C 2}=90 \mathrm{mT}$. A small hysteresis loop is seen at $H<H_{C 1}$.

Concluding this section, we have analyzed three contributions to the neutron scattering: nuclear one, magnetic one, and interference between them. The nuclear contribution is the biggest one. It gives the information about the structure of the inversed $\mathrm{PhC}$ and should be compared to the results obtained from microradian small-angle x-ray scattering experiment. It appears that the ultrasmall-angle diffraction of the synchrotron radiation is a much more effective tool for studying the structure of the opal-like structures due to its high brilliance and high resolution. On the other hand, although the magnetic and nuclear-magnetic interference contributions to the scattering are weak with a poor statistics, the polarized small-angle neutron diffraction remains irreplaceable in the study of the magnetic structures. Interference scattering in the recorded reflections gives the pattern behavior of the reversal magnetization process for the corresponding reflecting planes. The pure magnetic scattering of the corresponding Bragg peaks can be related to the complex distribution of the magnetic-flux lines in the geometrical network of the octahedral and tetrahedral particles; a certain set of which is the base of the structure element of the inverse opal photonic crystal. In this sense, the polarized small-angle neutron diffraction is a direct method for measuring this complex magnetic network that appeared in the course of the magnetization process of such inverse $\mathrm{PhC}$. Yet, a detailed analysis of these data is not a trivial task and lies beyond the scope of this paper.

\section{CONCLUDING REMARKS}

In conclusion, the structural and magnetic properties of nickel inverse opal photonic crystal have been studied on the mesoscopic and microscopic levels by different experimental techniques-XRD, SEM, microradian $\mathrm{x}$-rays scattering, and polarized SANS methods.

(1) It is established from the microradian small-angle $\mathrm{x}$-ray diffraction that nickel inverse opal photonic crystal repeats completely the structure of a matrix of a photonic crystal with minor alteration of a lattice constant, which is equal to $650 \pm 10 \mathrm{~nm}$. From the analysis of defects of the structure, it became obvious that inverted opal has divided into blocks of the face-centered-cubic structures with twinning planes (ratio between $A B C A B C$ and $A C B A C B$ phases is $4 / 5$ ).

(2) From the results of the wide-angle powder-diffraction experiment, one can conclude that the inverse $\mathrm{PhC}$ consists of $\mathrm{Ni}$ crystallites of the fcc structure with the lattice constant $3.52 \AA$. Most of the Ni crystallites are randomly oriented but up to $10 \%$ of its amount prefer (200) planes parallel to the $\mathrm{PhC}$ surface.

(3) The polarized SANS was used to detect the transformation of the magnetic structure under the applied field. The interference term gives the pattern behavior of the average magnetization projected onto the field axis. The patterns of hysteresis loops of the magnetization are different for different orientations of the magnetic field applied along two principal axes of the PhC: along the [2 $\overline{4} 2]$ and $[11 \overline{1}]$ axes. This is related to the geometrical shape of the inverse opal photonic crystal. The picture of the magnetization process can be studied in more details in the analysis of changes in the pure magnetic contribution of the corresponding Bragg peaks. In general, the experiment has demonstrated that the 3D geometrical shape of the structure leads to a complex distribution of the magnetization in the sample, which is far from being simple uniform.

\section{ACKNOWLEDGMENTS}

This work is supported in part by the RFBR (Project No. 08-03-00938) and the Goskonracts of the Russian Government under Grants No. 02.513.11.3186 and No. 02.513.11.3352. The beamtime at the DUBBLE beamline is kindly provided by the Netherlands Organisation for Scientific Research (NWO). We thank Kristina Kvashnina and Dirk Detollenaere from the DUBBLE beamline for their excellent support. We are grateful to Wim Bouwman for his help in conducting the small-angle $\mathrm{x}$-ray diffraction experiment. The PNPI team acknowledges GKSS for their hospitality. 
${ }^{1}$ M. Inoue, H. Uchida, K. Nishimura, and P. B. Lim, J. Mater. Chem. 16, 678 (2006).

${ }^{2}$ L. Sun, Y. Hao, C.-L. Chien, and P. C. Searson, IBM J. Res. Dev. 49, 79 (2005).

${ }^{3}$ E. Yablonovitch, Phys. Rev. Lett. 58, 2059 (1987).

${ }^{4}$ S. John, Phys. Rev. Lett. 58, 2486 (1987).

${ }^{5}$ M. Inoue, R. Fujikawa, A. Baryshev, A. Khanikaev, P. B. Lim, H. Uchida, O. Aktsipetrov, A. Fedyanin, T. Murzina, and A. Granovsky, J. Phys. D 39, R151 (2006).

${ }^{6}$ R. F. Wang, C. Nisoli, R. S. Freitas, J. Li, W. McConville, B. J. Cooley, M. S. Lund, N. Samarth, C. Leighton, V. H. Crespi, and P. Schiffer, Nature (London) 439, 303 (2006).

${ }^{7}$ M. N. Baibich, J. M. Broto, A. Fert, F. Nguyen Van Dau, F. Petroff, P. Etienne, G. Creuzet, A. Friederich, and J. Chazelas, Phys. Rev. Lett. 61, 2472 (1988).

${ }^{8}$ P. Grünberg, R. Schreiber, Y. Pang, M. B. Brodsky, and H. Sowers, Phys. Rev. Lett. 57, 2442 (1986).

${ }^{9}$ X. Xu, G. Friedman, K. D. Humfeld, S. A. Majetich, and S. A. Asher, Adv. Mater. 13, 1681 (2001).

${ }^{10}$ J. Ge, Y. Hu, M. Biasini, W. P. Beyermann, and Y. Yin, Angew. Chem. Int. Ed. 46, 4342 (2007).

${ }^{11}$ A. Stein, Microporous Mesoporous Mater. 44-45, 227 (2001).

${ }^{12}$ H. Yan, C. F. Blanford, B. T. Holland, M. Parent, W. H. Smyrl, and A. Stein, Adv. Mater. (Weinheim, Ger.) 11, 1003 (1999).

${ }^{13}$ L. Xu, W. L. Zhou, C. Frommen, R. H. Baughman, A. A. Zakhidov, L. Malkinski, J.-Q. Wang, and J. B. Wiley, Chem. Commun. (Cambridge) (2000) 997.

${ }^{14}$ P. N. Bartlett, P. R. Birkin, and M. A. Ghanem, Chem. Commun. (Cambridge) (2000) 1671.

${ }^{15}$ P. N. Bartlett, M. A. Ghanem, I. S. El Hallag, P. de Groot, and A. Zhukov, J. Mater. Chem. 13, 2596 (2003).

${ }^{16}$ K. S. Napolskii, A. Sinitskii, S. V. Grigoriev, N. A. Grigorieva, H. Eckerlebe, A. A. Eliseev, A. V. Lukashin, and Yu. D. Tretyakov, Physica B 397, 23 (2007).

${ }^{17}$ X. Yu, Y.-J. Lee, R. Furstenberg, J. O. White, and P. V. Braun,
Adv. Mater. (Weinheim, Ger.) 19, 1689 (2007).

${ }^{18}$ S. Fan, P. R. Villeneuve, and J. D. Joannopoulos, Phys. Rev. B 54, 11245 (1996).

${ }^{19}$ A. Moroz, Phys. Rev. Lett. 83, 5274 (1999).

${ }^{20}$ R. M. Amos, J. G. Rarity, P. R. Tapster, T. J. Shepherd, and S. C. Kitson, Phys. Rev. E 61, 2929 (2000).

${ }^{21}$ B. G. Prevo and O. D. Velev, Langmuir 20, 2099 (2004).

${ }^{22}$ A. S. Sinitskii, V. V. Abramova, T. V. Laptinskaya, and Yu. D. Tretyakov, Phys. Lett. A 366, 516 (2007).

${ }^{23}$ I. M. Sosnowska and M. Shiojiri, J. Electron Microsc. 48, 681 (1999).

${ }^{24}$ J. Zhu, M. Li, R. Rogers, W. Meyer, R. H. Ottewill, STS-73 Space Shuttle Crew, W. B. Russel, and P. M. Chaikin, Nature (London) 387, 883 (1997).

${ }^{25}$ Ch. Dux and H. Versmold, Phys. Rev. Lett. 78, 1811 (1997).

${ }^{26}$ W. K. Kegel and J. K. G. Dhont, J. Chem. Phys. 112, 3431 (2000).

${ }^{27}$ A. V. Petukhov, D. G. A. L. Aarts, I. P. Dolbnya, E. H. A. de Hoog, K. Kassapidou, G. J. Vroege, W. Bras, and H. N. W. Lekkerkerker, Phys. Rev. Lett. 88, 208301 (2002).

${ }^{28}$ H. Versmold, S. Musa, and A. Bierbaum, J. Chem. Phys. 116, 2658 (2002).

${ }^{29}$ A. V. Petukhov, J. H. J. Thijssen, D. C. 't Hart, A. Imhof, A. van Blaaderen, I. P. Dolbnya, A. Snigirev, A. Moussaïd, and I. Snigireva, J. Appl. Crystallogr. 39, 137 (2006).

${ }^{30}$ J. H. J. Thijssen, A. V. Petukhov, D. C. 't Hart, A. Imhof, C. H. M. van der Werf, R. E. I. Schropp, and A. van Blaaderen, Adv. Mater. (Weinheim, Ger.) 18, 1662 (2006).

${ }^{31}$ A. V. Petukhov, I. P. Dolbnya, D. G. A. L. Aarts, G. J. Vroege, and H. N. W. Lekkerkerker, Phys. Rev. Lett. 90, 028304 (2003).

${ }^{32}$ W. L. Vos, M. Megens, C. M. van Kats, and P. Bosecke, Langmuir 13, 6004 (1997).

${ }^{33}$ J. E. G. J. Wijnhoven, L. Bechger, and W. L. Vos, Chem. Mater. 13, 4486 (2001) 